

Revista Científica Mundo de la Investigación y el Conocimiento

Walter Alejandro Patiño Zambrano a ; Viviana Paola Patiño Zambrano ${ }^{\text {b; }}$ Robín Edison Cedeño Mero ${ }^{c}$; José Eduardo Cedeño Gilces ${ }^{d}$

Prevalencia de fisura labio palatina en niños menores de 1 año en la consulta de cirugía estética reconstructiva

Revista Científica Mundo de la Investigación y el Conocimiento. Vol. 2 núm., 1, febrero, ISSN: 2588-073X, 2018, pp. 674-683

DOI: $10.26820 /$ recimundo/2.1.2018.674-683

Editorial Saberes del Conocimiento

Recibido: 05/12/2017

Aceptado: 10/02/2018

a. Universidad de Guayaquil; alejo8585@ hotmail.com

b. Universidad de Guayaquil; viviana.patiñoz@ug.edu.ec

c. Especialista en Otorrinolaringología; med2008robin@gmail.com

d. Médico de la Universidad de Guayaquil; educedenomd@ hotmail.com 


\section{Prevalencia de fisura labio palatina en niños menores de 1 año en la consulta de cirugía estética reconstructiva}

Vol. 2, núm. 1., (2018)

Walter Alejandro Patiño Zambrano; Viviana Paola Patiño Zambrano; Robín Edison Cedeño

Mero; José Eduardo Cedeño Gilces

\section{RESUMEN}

En la siguiente investigación se aborda un estudio sobre la Fisura Labio Palatina (FLP), la cual es una de las malformaciones congénitas más frecuentes. Esta patología representa un problema de mucha trascendencia desde el punto de vista individual del niño afectado, ya que por su ubicación exige una atención rápida para facilitar al paciente la alimentación, fonación y en una forma sostenida la oclusión dentaria. En diversos estudios durante las últimas décadas expertos en el ámbito maxilofacial han coincidido en que la resolución del paladar hendido en pacientes durante el primer año de vida aporta mayores beneficios desde el punto de vista foniátrico y estético. Se pudo concluir que mientras más temprano se realice el tratamiento integral será mejor la imagen corporal y por ende su integración social, además de colaborar en su buena nutrición.

Palabras Claves: Fisura Labio Palatino, alimentación, foniátrico, estética e intervención quirúrgica. 


\section{Prevalencia de fisura labio palatina en niños menores de 1 año en la consulta de cirugía estética reconstructiva}

Vol. 2, núm. 1., (2018)

Walter Alejandro Patiño Zambrano; Viviana Paola Patiño Zambrano; Robín Edison Cedeño Mero; José Eduardo Cedeño Gilces

\section{ABSTRACT}

In the following investigation, a study on Palatal Lip Fissure (FLP) is studied, which is one of the most frequent congenital malformations. This pathology represents a problem of great importance from the individual point of view of the affected child, since its location requires rapid attention to facilitate the patient feeding, phonation and in a sustained dental occlusion. In several studies during the last decades experts in the maxillofacial field have agreed that the resolution of the cleft palate in patients during the first year of life brings greater benefits from the phonetic and aesthetic point of view. It was possible to conclude that the earlier the integral treatment is carried out, the better the body image and therefore its social integration, in addition to collaborating in its good nutrition.

Key Words: Cleft Palatal lip, feeding, phoniatrics, aesthetics and surgical intervention. 


\section{Prevalencia de fisura labio palatina en niños menores de 1 año en la consulta de cirugía estética reconstructiva}

Vol. 2, núm. 1., (2018)

Walter Alejandro Patiño Zambrano; Viviana Paola Patiño Zambrano; Robín Edison Cedeño

Mero; José Eduardo Cedeño Gilces

\section{Introducción.}

La Fisura Labio Palatina (FLP) es una de las malformaciones congénitas más frecuentes y se produce por una alteración en la fusión de los tejidos que darán origen al labio y al paladar, durante el desarrollo embrionario. El labio y paladar hendido pueden ocurrir juntos o separados. El labio hendido con o sin paladar hendido ocurre en 1:1000 nacidos mientras que el paladar hendido sólo ocurre en aproximadamente 1:2500 nacidos. El labio hendido (con o sin paladar hendido) es más común en el sexo masculino mientras el paladar hendido es más común en el sexo femenino (Bedón \& Villota, 2012).

El paladar fisurado está caracterizado por ser un defecto que afecta generalmente al labio superior cerca del 75\%-80\% de los casos, son con mayor frecuencia unilaterales siendo el lado izquierdo el más afectado (Zambrana, 2012). Las hendiduras orofaciales representan una gran proporción de defectos de nacimiento (congénitos) de causa multifactorial. Se ha pensado que tanto factores ambientales como genéticos contribuyen a su aparición, sin embargo, también se han propuesto varios factores tanto maternos, de género, étnicos entre otros. Por tanto, su etiología es compleja y multifactorial (España, 2015).

Esta patología representa un problema de mucha trascendencia desde el punto de vista individual del niño afectado, ya que por su ubicación exige una atención rápida para facilitar al paciente la alimentación, fonación y en una forma sostenida la oclusión dentaria. El cierre quirúrgico del paladar hendido o palatorrafia, tiene como objetivos devolver su estructura o conformación anatómica y funciones como deglución, fonación y masticación (Monserat \& al., 2000). 


\section{Prevalencia de fisura labio palatina en niños menores de 1 año en la consulta de cirugía estética reconstructiva}

Vol. 2, núm. 1., (2018)

Walter Alejandro Patiño Zambrano; Viviana Paola Patiño Zambrano; Robín Edison Cedeño Mero; José Eduardo Cedeño Gilces

La literatura coincide en que la reparación quirúrgica de un paciente con labio hendido no es una urgencia. En la actualidad la reparación primaria de labio hendido se realiza alrededor de los 3 meses de edad. Se recomienda que los niños sean mayores de 10 semanas de edad, peso de por lo menos $4.5 \mathrm{~kg}$ y valores de hemoglobina mayor a $10 \mathrm{~g} / \mathrm{dL}$. El paladar hendido y el maxilar se cierran al final del 1er año de vida. Con estos criterios se disminuyen los riesgos tanto anestésicos como quirúrgicos. Con los nuevos avances en las técnicas quirúrgicas, esta cirugía desarrollada incluso en edades tempranas puede ofrecer mejores resultados estéticos y funcionales, por otro lado, no se reporta mortalidad y un índice muy bajo de morbilidad (Alarcón, 2010, Abril-Junio ).

El tratamiento integral de las fisuras labiomaxilo-palatinas debe ser conducido por un equipo multidisciplinario formado por: cirujanos, otorrinolaringólogos, odontólogos, psicólogos, foniatras. Este equipo tiene que estar constituido por una unidad donde conjuntamente se programe cada paso del tratamiento y donde el paciente ingrese prácticamente al nacer (Bedón \& Villota, 2012).

\section{Materiales y métodos.}

Para este trabajo se recurrió al método de la investigación documental, la cual se basa en la recopilación de documentos, seleccionarlos, analizarlos; para luego presentar resultados coherentes. Se puede definir de la siguiente manera:

- Realiza un proceso de abstracción científica, generalizando sobre la base de lo fundamental 


\section{Prevalencia de fisura labio palatina en niños menores de 1 año en la consulta de cirugía estética reconstructiva}

Vol. 2, núm. 1., (2018) Walter Alejandro Patiño Zambrano; Viviana Paola Patiño Zambrano; Robín Edison Cedeño Mero; José Eduardo Cedeño Gilces

- Utiliza los procedimientos lógicos y mentales de toda investigación; tales como: análisis, síntesis, deducción, inducción, entre otros.

- Supone una recopilación adecuada de datos, que permiten redescubrir hechos, sugerir problemas, ubicar hacia otras fuentes de investigación, orientar formas para elaborar instrumentos de investigación y elaborar hipótesis (Rodriguez, 2013).

También se respalda el estudio con una investigación bibliográfica, que permite, entre otras cosas, evitar emprender investigaciones anteriormente ejecutadas, obtener conocimiento de experimentos previos para repetirlos en caso de ser necesario, buscar información sugerente, culminar investigaciones interrumpidas o incompletas, seleccionar los materiales y documentos para un marco teórico, entre otros propósitos (Rodriguez, 2013).

Con los conceptos expuestos se puede decir que en el presente estudio se utilizó como metodología una revisión bibliográfica documental no experimental, puesto que realizó una revisión y análisis de diferentes artículos médicos y de opinión referentes a la a prevalencia de fisura labio palatina en niños menores de 1 año, de esta manera se pudo abordar sus causas, tratamiento y consecuencias; además de poder aportar recomendaciones.

\section{Resultados.}

En diversos estudios durante las últimas décadas expertos en el ámbito maxilofacial han coincidido en que la resolución del paladar hendido en pacientes durante el primer año de vida aporta mayores beneficios desde el punto de vista foniátrico y estético; sin embargo, existen vertientes las cuales indican que es pertinente realizar la cirugía durante la etapa preescolar para 


\section{Prevalencia de fisura labio palatina en niños menores de 1 año en la consulta de cirugía estética reconstructiva}

Vol. 2, núm. 1., (2018)

Walter Alejandro Patiño Zambrano; Viviana Paola Patiño Zambrano; Robín Edison Cedeño Mero; José Eduardo Cedeño Gilces

un mejor desarrollo odontológico. En dichos estudios los investigadores han obtenido los siguientes resultados:

- El tratamiento quirúrgico primario busca lograr un cierre óptimo, una perfecta función y buen resultado estético, por lo cual es necesario que al paciente se le refiera a la unidad especializada prácticamente desde los primeros días de vida. Se requieren un mínimo de 4 eventos quirúrgicos por caso. El primero a los tres meses de edad para cierre de labio y plastia de punta nasal; el segundo entre los 12 y 18 meses de edad para el cierre del paladar y faringoplastia; el tercero entre los 6 y 8 años de edad con injerto óseo alveolar; el cuarto se efectúa después de los 14 años y es la cirugía estética facial (Lombardo, 2017).

- En estudios anteriores se observó hipodesarrollo de la cara, atrofia del reborde alveolar, y en general desarrollo anormal del maxilar superior como consecuencia del tratamiento quirúrgico en épocas tempranas de la vida del paciente. Por este motivo los odontólogos proponen el tratamiento ortodóntico después de la erupción de los segundos molares temporales, también el tratamiento ortopédico precoz antes y después de la palatorrafia (Bedón \& Villota, 2012).

- Presentar una hendidura facial impone una diferencia visual evidente, esto en la mayoría de los casos produce un impacto profundo en las relaciones interpersonales del afectado, su interacción con la sociedad, su desempeño laboral, su autoestima y lo más importante en su calidad de vida (España, 2015). 


\section{Prevalencia de fisura labio palatina en niños menores de 1 año en la consulta de cirugía estética reconstructiva}

Vol. 2, núm. 1., (2018) Walter Alejandro Patiño Zambrano; Viviana Paola Patiño Zambrano; Robín Edison Cedeño Mero; José Eduardo Cedeño Gilces

\section{Conclusiones.}

- El labio y paladar hendido como malformación congénita continúan siendo un foco de investigación en el mundo por su alta incidencia en la población general

- Los pacientes con labio hendido presentan una deficiencia funcional grave del lenguaje, la masticación y la deglución.

- Los pacientes con labio y paladar hendido poseen tendencia a bajo peso debido a que se alimentan con bastante dificultad, ya que no es posible crear un cierre hermético con la boca y por esto pueden presentar retraso en el desarrollo y crecimiento (España, 2015).

- En diversos estudios se puede evidenciar que el componente genético se encuentra fuertemente ligado, ya que el antecedente familiar con labio y paladar hendido aumenta el riesgo de heredarle de 4 a 20\%. (Lombardo, 2017).

- El sexo masculino es más susceptible a presentar hendidura labial aislada y hendidura de labio y paladar (Bedón \& Villota, 2012).

- El pronóstico depende totalmente de la atención que se brinde al paciente por lo que mientras más precoz sea el abordaje mejor resultados se obtendrán.

- Mientras más temprano se realice el tratamiento integral será mejor la imagen corporal y por ende su integración social (Corbo, 2001).

\section{Recomendaciones.}

- La intervención del pediatra en el manejo del paciente con labio y paladar hendido es fundamental ya que es quién tiene el primer contacto con estos pacientes, desde la 


\section{Prevalencia de fisura labio palatina en niños menores de 1 año en la consulta de cirugía estética reconstructiva}

Vol. 2, núm. 1., (2018)

Walter Alejandro Patiño Zambrano; Viviana Paola Patiño Zambrano; Robín Edison Cedeño Mero; José Eduardo Cedeño Gilces

atención del recién nacido en las unidades quirúrgicas o durante las primeras semanas de vida en la consulta pediátrica (Lombardo, 2017).

- Es fundamental la atención multidisciplinaria la cual no debe excluir nunca la atención psicosocial ya que estos casos pacientes tienen a padecer repercusiones en dicho ámbito sobre todo en las etapas escolar y adolescente.

- De igual manera se recomienda atención precoz y evaluación para evitar las complicaciones de esta enfermedad.

\section{Bibliografía.}

Alarcón, J. (2010, Abril-Junio ). Labio y paladar hendido. Anestesiología en pediatría Vol. 33. Supl. 1, recuperado de: http://www.medigraphic.com/pdfs/rma/cma-2010/cmas 101r.pdf, S76-S78.

Bedón, M., \& Villota, L. (2012). Labio y paladar hendido: Tendencias actuales en el manejo exitoso . Archivos de Medicina $(\mathrm{Col})$, vol. 12, núm. 1, recuperado de: http://www.redalyc.org/articulo.oa? $i d=273824148010,107-119$.

Corbo, M. (2001). Labio y palada fisurados: Aspectos generales que se deben conocer en la atención primaria de salud. Rev Cubana Med Gen Integr, recuperado de: http://bvs.sld.cu/revistas/mgi/vol17_4_01/mgi11401.pdf, 17 (4): 379-85.

España, L. (2015). Caracterización de pacientes con labio y paladar hendido . Fundación Operación Sonrisa Guatemala, recuperado de: http://recursosbiblio.url.edu.gt/tesisjcem/2015/09/03/Espana-Lilly.pdf .

Lombardo, A. (2017). La intervención del pediatra en el niño con labio y paladar hendido. • LOMBARDO A. LA INTERVENCIÓN DEL PEDIATRA EN EL NIActa Pediatr Mex, recuerado de : http://www.medigraphic.com/pdfs/actpedmex/apm-2017/apm174f.pdf, 38 (4): $267-273$.

Monserat, \& al., e. (2000). Labio y paladar hendido. Acta odontol. venez v.38 n.3. recuperado de:

http://www.scielo.org.ve/scielo.php? pid=S000163652000000300004\&script=sci_arttext\&tlng=pt. 


\section{Prevalencia de fisura labio palatina en niños menores de 1 año en la consulta de cirugía estética reconstructiva}

Vol. 2, núm. 1., (2018)

Walter Alejandro Patiño Zambrano; Viviana Paola Patiño Zambrano; Robín Edison Cedeño

Mero; José Eduardo Cedeño Gilces

Rodriguez, M. (2013). Acerca de la investigación bibliográfica y documental. recuperado de: http://guiadetesis. wordpress. com/2013/08/19/acerca-de-la-investigacion-bibliograficaydocumental.

Zambrana, O. (2012). Fisura labio palatina (FLP). Rev. Act. Clin. Med, recuperado de: http://www.revistasbolivianas.org.bo/scielo.php?script $=$ sci_arttext\&pid=S2304$37682012000100008 \& \operatorname{lng}=e s \& n r m=i s o$ 